\title{
Terahertz near-field imaging of surface plasmon waves in graphene structures
}

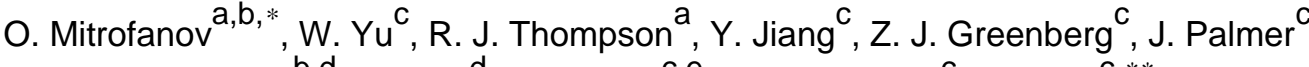 \\ I. Brener ${ }^{b, d}$, W. Pan ${ }^{d}$, C. Berger ${ }^{c, e}$, W. A. de Heer ${ }^{c}$, Z. Jiang ${ }^{c, * *}$ \\ ${ }^{\mathrm{a}}$ Electronic and Electrical Engineering, University College London, London WC1E 7JE, United Kingdom \\ ${ }^{b}$ Center for Integrated Nanotechnologies, Sandia National Laboratories, Albuquerque, New Mexico 87185, U.S.A. \\ c School of Physics, Georgia Institute of Technology, Atlanta, Georgia 30332, U.S.A. \\ d Sandia National Laboratories, Albuquerque, New Mexico 87185, U.S.A. \\ e CNRS/Institut Néel, BP166, 38042 Grenoble, France
}

\begin{abstract}
We introduce a near-field scanning probe terahertz ( $\mathrm{THz}$ ) microscopy technique for probing surface plasmon waves on graphene. Based on $\mathrm{THz}$ time-domain spectroscopy method, this near-field imaging approach is well suited for studying the excitation and evolution of $\mathrm{THz}$ plasmon waves on graphene as well as for mapping of graphene properties at $\mathrm{THz}$ frequencies on the sub-wavelength scale.
\end{abstract}

Keywords: E. THz near-field microscopy; D. Surface plasmons; A. Epitaxial graphene.

\section{Introduction}

Graphene plasmons, the collective oscillations of Dirac fermions enabling strong enhancement of lightmatter interaction, have recently attracted a great deal of attention (for a recent review, see Ref. [1]). It has been shown that surface plasmons can be confined in graphene nanostructures with a characteristic dimension more than 100 times smaller than the wavelength of incident light [2,3]. Graphene plasmons are highly tunable; the plasmon frequency depends on the carrier density and doping [4-6], the characteristic dimension (for graphene structures) [4, 5, 7], the number of stacked layers [5], the packing density of graphene structures [8], the substrate phonon modes [9, 10], and the applied magnetic field [3, $7,11]$. Graphene plasmons can be excited and probed locally using a scattering-type scanning near-field optical microscope with a focused infrared beam $[12,13]$. Such a high-resolution near-field technique has created great excitement in the field. The spectral range of these studies $\left(600-2500 \mathrm{~cm}^{-1}\right)$ was however limited by the mid-infrared light source [14].

In the terahertz $(\mathrm{THz})$ spectral range $\left(10-100 \mathrm{~cm}^{-1}\right)$ graphene also exhibits rich physics and great application potential [15-17]. THz spectroscopy and imaging techniques have been used to study the $\mathrm{THz}$ conductivity of graphene [18-20], enabling non-contact characterization of graphene and providing direct information about its doping level and uniformity. These far-field methods however provide only limited information about plasmon excitations, which are localized at the surface. In this Communication, we introduce a near-field scanning probe $\mathrm{THz}$ microscopy technique for probing graphene plasmons at $\mathrm{THz}$ frequencies. Based on time-domain analysis, this technique is particularly suited for studies of excitation and propagation of surface waves [21].

\section{Material and methods}

Graphene is expected to support THz surface plasmon waves. The surface waves can be excited by an incident plane wave at the edges of graphene pattern [21], similar to the excitation of surface waves at the edges of metallic films deposited on dielectric substrates [22, 23]. Graphene ribbon arrays on the

\footnotetext{
${ }^{*}$ Corresponding author. Tel: (O.M.)+44 (0)20 76793128.

${ }^{* *}$ Corresponding author. Tel: (Z.J.)+1 4043853906.

E-mail addresses: o.mitrofanov@ucl.ac.uk (O.M.), zhigang.jiang@physics.gatech.edu (Z.J.).
} 
other hand can be used for efficient excitation of confined plasmon modes by an incident plane wave [3, 4], similar to periodic metallic structures (arrays of ribbons or corrugations).

Unlike the metallic films however, graphene is highly transparent to the incident electromagnetic wave. The transmission coefficient for a graphene layer at $\mathrm{THz}$ frequencies can be reduced only to the level of $60-70 \%$, even for the highest achievable carrier densities [18-21]. This presents a challenge for observation and investigations of surface plasmon waves in graphene structures: the incident $\mathrm{THz}$ wave typically dominates the signal in the region where the confined plasmon modes and surface plasmon waves are excited.

Studies of surface plasmons in graphene therefore require both local (near-field) probing at the surface and a method for distinguishing surface plasmon waves from the incident wave. In this work, we will address the questions of excitation and detection of $\mathrm{THz}$ surface plasmon waves in patterned graphene structures illuminated by a plane incident wave. In our analysis of $\mathrm{THz}$ surface waves, we take advantage of the temporal resolution provided by the $\mathrm{THz}$ time-domain spectroscopy technique. Combined with near-field scanning probe THz microscopy, this method allows detecting temporal evolution of the electric field distribution over the surface, in which surface wave signatures become apparent [23].

\subsection{Epitaxial graphene on silicon carbide}

The epitaxial graphene studied in this work was grown on silicon carbide ( $\mathrm{SiC}$ ) substrate using the confinement controlled sublimation method [24]. Generally speaking, there are two principally different types of epitaxial graphene forming on the Si- or C-terminated face of $\mathrm{SiC}$, respectively. The growth on the Si-terminated face has a better layer control and the material consists of mono- and bi-layers of graphene but with a relatively high carrier concentration due to the charge transfer from SiC [25]. Graphene grown on the C-terminated face however is usually multilayered. Although the first few layers of graphene close to the interface with $\mathrm{SiC}$ are still highly doped (as that on the Si-terminated face), the subsequent top layers are practically charge neutral and electrically decoupled from each other behaving like monolayer graphene [26, 27]. The Fermi energy of such quasi-neutral top layers of graphene is typically $E_{F}<20 \mathrm{meV}[3,27]$, while $E_{F}>200 \mathrm{meV}$ for the highly doped layers close to the interface [25].

The epitaxial graphene samples were patterned into mesas and ribbon arrays via standard electronbeam lithography, followed by oxygen plasma etching and high vacuum annealing. The vacuum annealing process is crucial for removing the chemical residue left on graphene surface after lithography. The annealing was conducted in high vacuum $\left(<5 \times 10^{-5} \mathrm{mTorr}\right)$ at $600{ }^{\circ} \mathrm{C}$ for 2 hours. Cyclotron resonance measurements reveal that the vacuum annealed top graphene layers grown on the $\mathrm{C}$ terminated face recover their low density and high mobility, $E_{F} \approx 17 \mathrm{meV}$ and $\mu>50,000 \mathrm{~cm}^{2} V^{-1} \mathrm{~s}^{-1}$ [3], similar to the values observed in as-grown samples.

\subsection{THz near-field microscopy and its application on metallic surfaces}

The near-field probe employed in this study is an integrated sub-wavelength aperture probe, which detects $\mathrm{THz}$ waves through a $10 \mu \mathrm{m}$ input aperture in a thin gold screen [22]. The probe senses the $\mathrm{THz}$ field polarized in the plane of the metallic screen as well as the field normal to the screen $[28,29]$. The probe is positioned approximately $2-5 \mu \mathrm{m}$ from the sample surface and the sample is illuminated by a $\mathrm{THz}$ beam from the substrate side (as shown in Fig. 1(a)). In this configuration, the probe can detect both the incident $\mathrm{THz}$ wave and excited surface waves [23, 29]. For the excitation, we use short $\mathrm{THz}$ pulses ( 2 ps) with the spectrum covering the frequency range from 0.5 to $2.5 \mathrm{THz}$. Before discussing $\mathrm{THz}$ surface plasmon waves in graphene, we briefly examine $\mathrm{THz}$ surface plasmon signatures on metallic surfaces.

Consider a metallic structure, for example a bow-tie antenna, on a dielectric substrate illuminated by a plane wave at normal incidence from the substrate side, as illustrated in Fig. 1(a). We use a large diameter $\mathrm{THz}$ waveguide with a well-defined beam profile for illumination of the antenna. The waveguide also ensures that the incident wave is described by a narrow distribution of wave-vectors $\left(\left|k_{x}\right|<0.1 k_{z}\right)$ centered around $k_{x}=0$.

The incident $\mathrm{THz}$ wave induces charges at the edges of the bow-tie antenna. The electric field distribution as a result has regions of field concentration near the antenna edges, where the field 

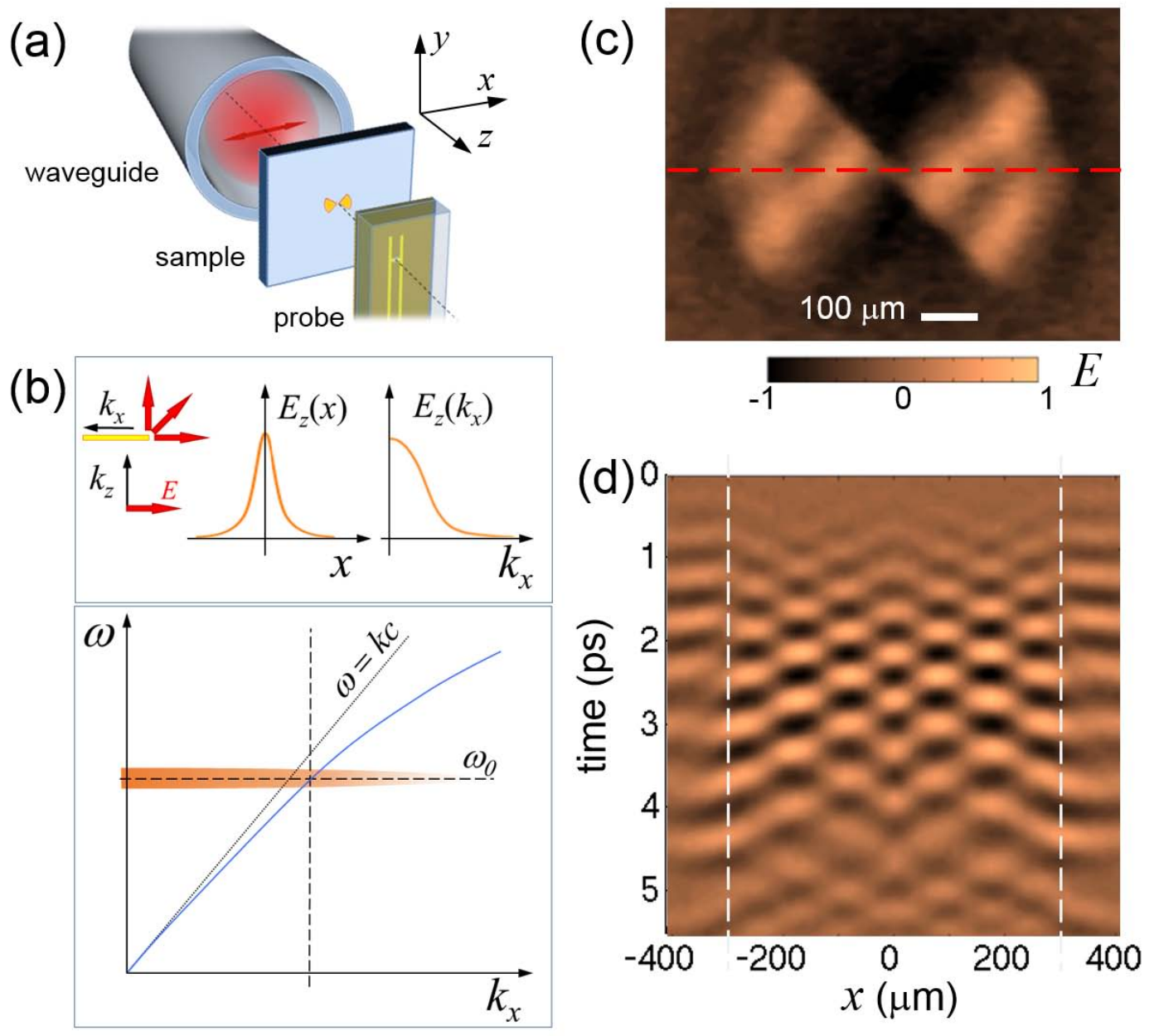

Fig. 1. Near-field microscopy system for probing THz surface plasmon waves. (a) Schematic diagram displaying a hollow $\mathrm{THz}$ waveguide, a sample with a metallic structure placed at the waveguide center, and the integrated $\mathrm{THz}$ near-field probe. (b) Mechanism of surface wave excitation at the edges of a metallic structure. The upper panel shows the spatial distribution and its angular spectrum for the $E_{z}$-component induced at the edge. The lower panel shows the dispersion relation for the surface wave (blue line) in a similar $k_{x}$ range as for the angular spectrum $E_{z}\left(k_{x}\right)$ in the upper panel. The black dotted line indicates the plane wave dispersion $\omega=k c$ for reference. (c) THz electric field distribution on a metallic bow-tie antenna deposited on a GaAs substrate. The snapshot corresponds to $t=1.16 \mathrm{ps}$. (d) A space-time map of the electric field near the antenna surface during the excitation of the antenna by a $\mathrm{THz}$ pulse. Data were taken along the red dashed line in (c). White dashed lines mark the location of the bow-tie edges.

component perpendicular to the surface, $E_{z}$ is created in addition to the $E_{x}$-component of the incident field (Fig. 1(b)). The $E_{z}$-component starts propagating away from the edge as a surface wave $[23,28]$.

To understand how the incident $\mathrm{THz}$ wave with $k_{x}=0$ couples into the surface wave, it is useful to consider the angular spectrum representation of the induced $E_{z}$-component near the edge. This field is localized at the edge and thus it contains a broad distribution of wave-vectors $k_{x}$ (as illustrated in the upper panel of Fig. 1(b)). For any frequency of the incident wave $\omega_{0}$, there is a component in the angular spectrum of $E_{z}$ that matches the transverse component $k_{x}$ required for launching a surface wave (lower panel of Fig. 1(b)). Therefore, the illumination of a metallic structure by a plane wave at normal incidence causes excitation of surface waves at the structure edges.

The process of excitation and propagation of $\mathrm{THz}$ surface waves at the edges of a metallic bow-tie antenna was studied in details by Mueckstein et al. [23]. Figure 1(c) shows an example of spatial distribution of the electric field on the antenna surface. The antenna surface becomes a resonator for the surface waves that move from one side of the antenna to the other. It is important to note that this $\mathrm{THz}$ image shows the electric field distribution on an opaque metallic surface illuminated from the substrate side. Therefore, only the surface waves contribute to the detected field over the antenna area [29]. 
The surface wave nature of the detected field is verified in its evolution displayed as a space-time map in Fig. 1(d). Here, the space-time map plots the field along the antenna length (marked by the red dashed line in Fig 1(c)) during the excitation by a short $\mathrm{THz}$ pulse and several picoseconds after it. The map shows that the field over the antenna area originates from the edges (marked by the white dashed lines). Note that the incident plane wave and the surface waves exhibit distinctive patterns in the space-time map. The phase of the incident plane wave is constant for any position $x$, thus it is represented by horizontal 'fringes' in the map (outside the region between the dashed lines). The phase of the surface wave on the other hand changes with $x$ according to the phase velocity relationship and it forms tilted 'fringes' in the map (in the region between the dashed lines).

The space-time map also illustrates that the field pattern on the antenna surface changes over time. At the beginning of excitation, the field is present only near the edges of the antenna. As time elapses the surface waves spread over the entire area of the antenna and a standing wave pattern forms. After the incident pulse passes, the surface waves remain on the antenna surface for several picoseconds, decreasing in amplitude over time and eventually extinguish.

\section{Results and discussion}

\subsection{THz near-field imaging of graphene bow-tie}

A bow-tie antenna made of monolayer epitaxial graphene grown on the Si-terminated face of SiC is
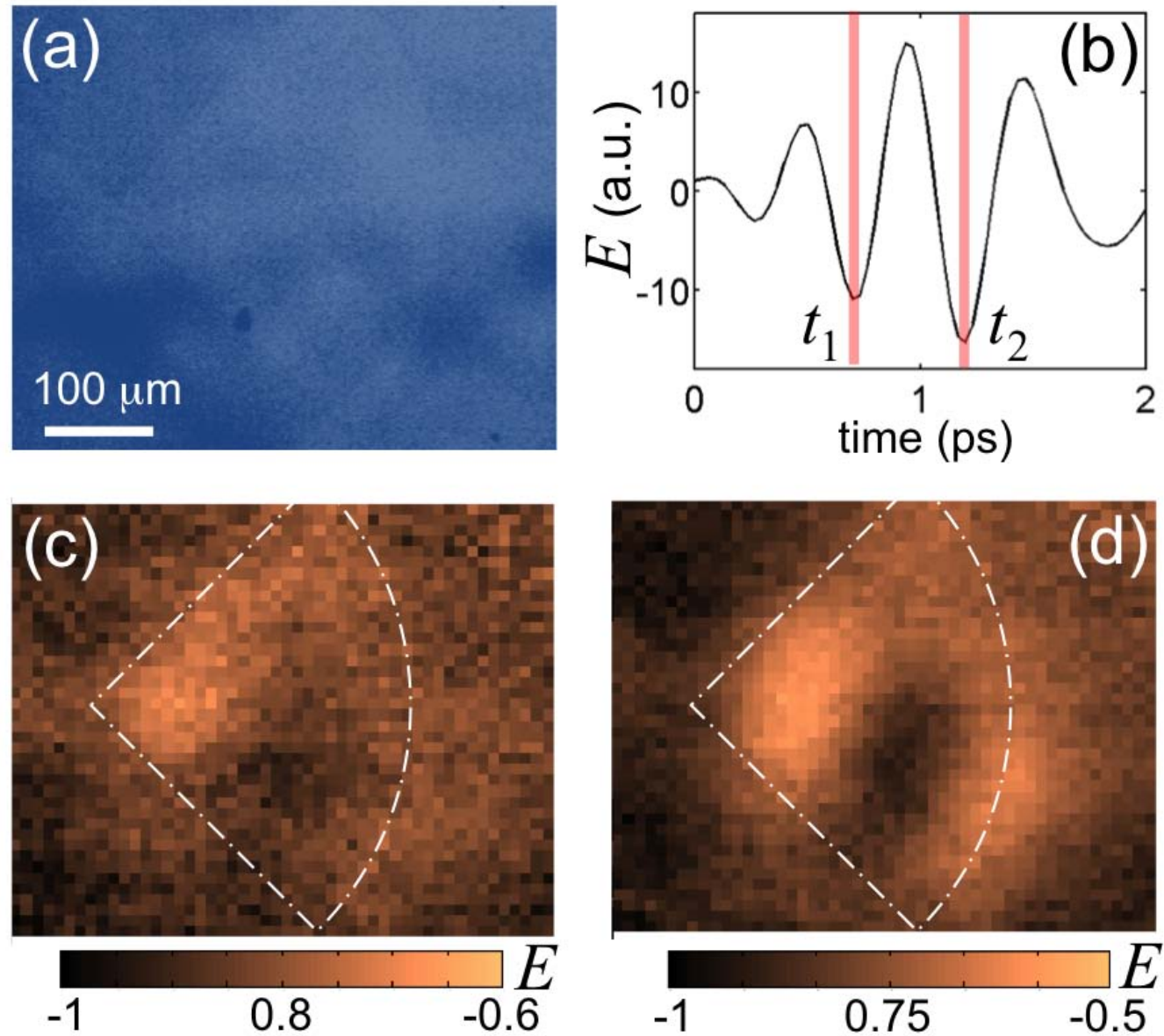

Fig. 2. Graphene bow-tie antenna patterned on SiC substrate. (a) Optical image of the right-hand side of the bow-tie. (b) Waveform of the incident THz pulse $E_{\text {inc }}(t)$. (c,d) THz near-field images of the bow-tie area in (a) captured at $t_{1}=0.67 \mathrm{ps}$ (c) and $t_{2}=1.13 \mathrm{ps}(\mathrm{d})$. The color scales are normalized to the field values $E_{\text {inc }}\left(t_{1}\right)$ and $E_{\text {inc }}\left(t_{2}\right)$, respectively. 
studied using the same THz near-field microscopy technique (Fig. 1(a)). The multilayer graphene that simultaneously grows on the C-terminated face, is removed by oxygen plasma etching. The monolayer graphene on the Si-terminated face is identified by Raman spectroscopy and confirmed by transport measurements on samples grown in the same condition. The right-hand side of the bow-tie structure is shown in the optical image in Fig. 2(a). The bow-tie geometry and dimensions are chosen to be the same as for the metallic bow-tie in Fig. 1(c). As in the experimental schematic shown in Fig. 1(a), the bow-tie is illuminated by the $\mathrm{THz}$ pulse (Fig. 2(b)) incident from the substrate side.

Two THz images acquired at two moments, $t_{1}$ and $t_{2}$, are shown in Figs. 2(c) and 2(d). In Fig. 2(c), the antenna makes only small impact on the uniform distribution of the electric field. Attenuation of the $\mathrm{THz}$ wave by graphene is barely noticeable. The image however shows variation of the field amplitude over the antenna area, similar to the surface wave interference pattern in Fig. 1(c). This pattern becomes more pronounced in Fig. 2(d) at $t_{2}=t_{1}+T$, where $T$ is approximately the period of the $\mathrm{THz}$ wave, indicating that more energy is converted from the incident plane wave into the surface wave.

The surface wave pattern observed on the graphene bow-tie remarkably replicates the pattern formed on the metallic bow-tie, despite the fact that the metallic layer is opaque for the incident $\mathrm{THz}$ wave, whereas the graphene layer is transparent. We note that in the case of graphene, the pattern is superimposed over the incident wave field, which is practically uniform in the $x y$-plane, while in the metallic bow-tie case, the pattern is formed in the 'shadow' area of the antenna, where the incident wave field is zero.

\subsection{THz near-field imaging of graphene ribbons}

The similarity between the patterns observed on the metallic and graphene bow-ties indicates that the corresponding surface waves are weakly confined as in the case of metallic surfaces [28]. Ribbon arrays on the other hand are predicted to support strongly confined plasmon modes, with the wave-vector several times larger than the free-space wave-vector. To investigate confined plasmon modes we now consider samples with periodic structures on the sub-wavelength scale.

Figure 3 shows a sample, which contains arrays of graphene ribbons oriented parallel and perpendicular to the electric field vector of the incident wave and two areas of continuous graphene, arranged as four quadrants. In this experiment, we use multilayer epitaxial graphene grown on the Cterminated face of $\mathrm{SiC}$. Ellipsometry measurements show that the sample consists of 10 graphene layers. The ribbons in both arrays are $W=4 \mu \mathrm{m}$ in width and the spacing between the adjacent ribbons is $4 \mu \mathrm{m}$. The frequency of the confined plasmon mode in ribbons is given by [3]

$$
\hbar \omega_{p l}=\sqrt{\frac{e^{2} E_{F}}{2 \varepsilon_{0} \varepsilon W}},
$$
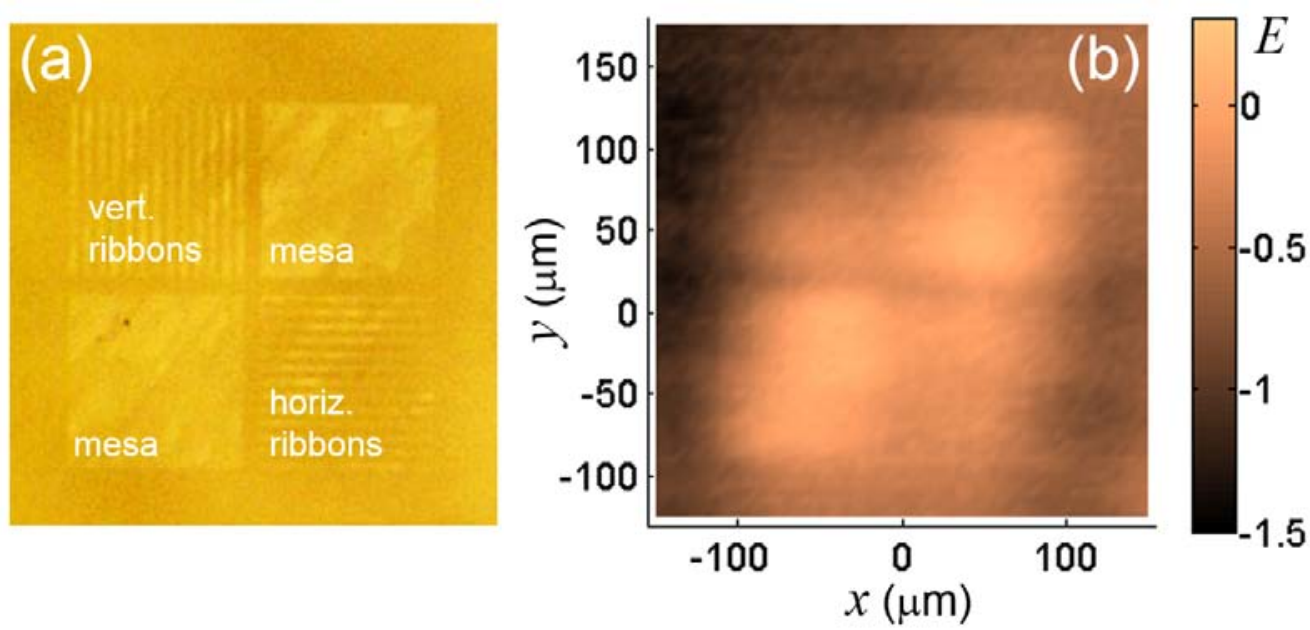

Fig. 3. THz near-field image of multilayer graphene (10-layer) mesas and ribbon arrays: (a) optical image and (b) $\mathrm{THz}$ transmission image of the same sample area. The size of the mesas is $100 \mu \mathrm{m}$ by $100 \mu \mathrm{m}$; the ribbons are $100 \mu \mathrm{m}$ in length and $4 \mu \mathrm{m}$ in width, and the spacing between the adjacent ribbons is $4 \mu \mathrm{m}$. 
where $\hbar$ is the reduced Planck's constant, $e$ is the electron charge, $\varepsilon_{0}$ is the vacuum permittivity, and $\varepsilon=\left(\varepsilon_{\text {SiC }}+1\right) / 2 \approx 5$ is the relative permittivity of epitaxial graphene. Taking $E_{F}=215 \mathrm{meV}$ [25] for the highly doped graphene layers near the interface with $\mathrm{SiC}$, we expect $\omega_{p l} / 2 \pi \approx 2.4 \mathrm{THz}$, while $\omega_{p l} / 2 \pi \approx 0.67 \mathrm{THz}$ for the quasi-neutral top layers where $E_{F}=17 \mathrm{meV}$ [3]. These frequencies fall in the spectral range of our measurement (0.5-2.5 THz).

In our experiment, we find that the detected $\mathrm{THz}$ field is noticeably reduced in the areas of all four quadrants. The continuous graphene mesas show lower transmission compared with the arrays, consistent with the reduced graphene area in the array. It is unlikely that the confined plasmons are excited in these arrays as their response to the incident $\mathrm{THz}$ pulse does not show a significant difference for both orientations of the ribbons. Arrays of $200 \mathrm{~nm}$-wide graphene ribbons also exhibit no effect of the ribbon orientation in our previous work [21].

A possible explanation for not being able to observe the confined plasmon mode in Fig. 3 is the charge inhomogeneity of the sample, particularly in the highly doped graphene layers close to the interface with SiC. While the near-field probe with a $10 \mu \mathrm{m}$ aperture does not resolve individual ribbons in Fig. 3, it shows that the transmission coefficient varies considerably within each $100 \mu \mathrm{m} \times 100 \mu \mathrm{m}$ quadrant. A similar variation of the local transmission properties has also been observed in other multilayer epitaxial graphene samples on SiC [21]. One can attribute this variation to the non-uniform electron density in graphene, caused potentially by the substrate. The electron density affects the dispersion relationship of surface plasmons and thus the non-uniformity is likely to scatter surface plasmon waves.

Arrays of $1 \mu \mathrm{m}$-wide graphene ribbons however exhibit a noticeable change in the transmission properties for the two orientations, parallel and perpendicular to the electric field vector. The ribbon arrays were fabricated from multilayer (10-layer) epitaxial graphene grown on the C-terminated face of SiC. The
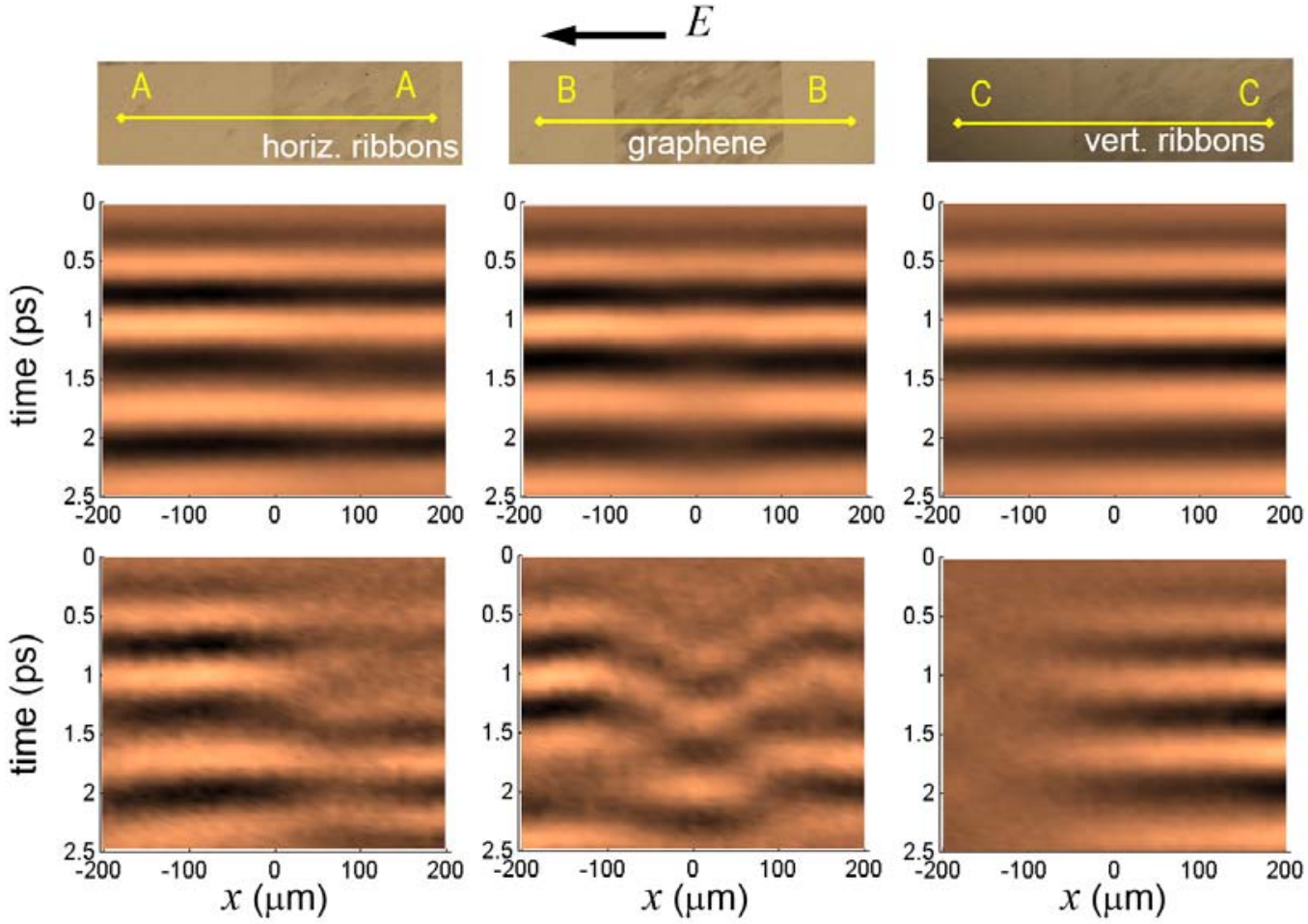

Fig. 4. Near-field THz space-time maps measured for a $200 \mu \mathrm{m} \times 200 \mu \mathrm{m}$ graphene mesa (middle) and arrays of $1 \mu \mathrm{m}$ wide graphene ribbons oriented parallel (left) and perpendicular (right) to the electric field polarization. The space-time maps were obtained within several microns from the sample surface. The top row shows the optical images of the samples; the middle row shows the detected $\mathrm{THz}$ field $E(x, t)$; and the bottom row shows $E(x, t)-\alpha \times E_{\text {inc }}(t)$, to emphasize the difference in electric field between the graphene pattern and the bare substrate. Here, $\alpha=0.73$ for horizontal ribbons (left), $\alpha=0.8$ for graphene mesa (middle), $\alpha=1$ for vertical ribbons (right), and $E_{\text {inc }}(t)$ is a waveform of the reference $\mathrm{THz}$ pulse taken on bare $\mathrm{SiC}$ substrate without graphene. 
ribbons are $200 \mu \mathrm{m}$ in length and $1 \mu \mathrm{m}$ in width, and the spacing between the adjacent ribbons is $1.2 \mu \mathrm{m}$. Figure 4 illustrates the orientation dependence by comparing $\mathrm{THz}$ space-time maps for three patterns fabricated on the same substrate. The left and right columns display experimental results for the ribbon arrays oriented parallel (left) and perpendicular (right) to the electric field, while the middle column shows the map of continuous graphene $(200 \mu \mathrm{m} \times 200 \mu \mathrm{m}$ square). Although the detected field, $E(x, t)$, is dominated by the incident plane wave (middle row of Fig. 4), there are noticeable changes in the field amplitude in the graphene regions.

To display the changes, we subtract the incident wave from the space-time maps in the bottom row of Fig. 4. For the ribbons parallel to the field, the incident field is scaled by a factor of 0.73 and subtracted from the detected field, $E(x, t)-0.73 \times E_{i n c}(t)$ to remove the field transmitted through the ribbon area (shown as vanishing fringes). The subtraction illustrates that the transmission through the horizontal ribbons is reduced by $\sim 27 \%$ compared to the bare SiC substrate. For the ribbons oriented perpendicular to the field, $E(x, t)-E_{\text {inc }}(t)$ plotted. In this case, we find surprisingly that more transmission occurs through the ribbon area (shown as remaining fringes) after subtracting the waveform of the incident $\mathrm{THz}$ pulse through the substrate. Quantitative analysis shows that the observed transmission is increased by $\sim 50 \%$ compared to the bare SiC substrate.

In addition, we notice that the subtracted maps in the left and right columns of Fig. 4 show no surface wave patterns in the ribbon array region. Similar background subtraction conducted for the space-time map measured over the graphene mesa (middle column) however reveals the signature of surface waves: the phase of the wave present in the mesa area changes with $x$, indicating that the surface waves originate from the edges. The lack of surface waves in graphene ribbon arrays can be caused by several factors: the excitation of surface waves at the end of the ribbon is likely to be less efficient compared to the large-area mesa; the (rough) ribbon edges can also provide additional absorption or scattering for a wave propagating along the ribbon.

The increase in transmission coefficient for the area with ribbons oriented perpendicular to the electric field compared to the bare $\mathrm{SiC}$ substrate requires a more detailed consideration. One possible interpretation is that it is due to the impedance matching provided by the graphene ribbon array. The THz wave incident on the SiC-air interface experiences significant partial reflection because of the large refractive index contrast $\left(n_{\text {sic }} \approx 3.0\right)$. The reflection coefficient can be reduced if graphene acts as an impedance matching layer. For example, it was reported recently that a continuous graphene layer can exhibit complete elimination of reflection from the quartz-air and silicon-air interfaces [30]. In the case of the ribbon array, the impedance matching can occur if the reflected waves from the Sic-air (between adjacent ribbons) and SiC-graphene-air interface have opposite polarities, producing a destructive interference and therefore minimizing the reflected wave amplitude. This effect should not be frequency selective, but dependent on the filling ratio of the array and the Fermi energy. The latter affects the conductivity of graphene. The impedance matching explanation is consistent with the lack of the surface waves originating from the ribbon array edges. Further finite-element simulations are needed to explain why the orientation dependent effect is absent in the $4 \mu \mathrm{m}$-wide and $200 \mathrm{~nm}$-wide ribbon arrays with a similar graphene filling ratio $(\sim 50 \%)$.

Finally, we examine the role of the confined surface plasmons in the effect of enhanced transmission through the ribbons oriented perpendicular to the electric field. The high-mobility quasi-neutral top layers in graphene ribbons on the $\mathrm{C}$-face of $\mathrm{SiC}$ are expected to exhibit a resonance frequency of $\omega_{p l} / 2 \pi \approx 1.3 \mathrm{THz}$ (from Eq. (1)) centered in our spectral range (0.5-2.5 THz). Our experiment however shows no signatures of the surface plasmon mode, e.g., a phase shift in the detected field or induced oscillations following the incident pulse. It suggests that the response of the ribbons is likely to be dominated by the highly doped layers of graphene close to the interface with SiC. The corresponding resonance frequency, $\omega_{p l} / 2 \pi \approx 4.8 \mathrm{THz}$, is beyond the spectral range of our technique. Further experimental work is needed to separate the contributions of the confined plasmons in the top and the interfacial layers and understand their effects on the observed enhanced transmission. 


\section{Conclusions}

We have performed a THz near-field microscopy study of epitaxial graphene structures (bow-ties, mesas, and ribbons) and observed surface plasmon waves excited at the edges of graphene structures (similar to the surface waves excited at metallic edges). Near-field images show that the $\mathrm{THz}$ absorption of graphene varies on a scale smaller than the wavelength and the Fresnel reflection at the SiC substrate surface is modified by the presence of graphene ribbons, leading to either reduced or enhanced transmission of $\mathrm{THz}$ waves depending on the orientation of the ribbons with respect to the electric filed and the ribbon width. The observations illustrate intriguing properties of graphene, which hold promise for new applications in $\mathrm{THz}$ spectroscopy, sensing, imaging, and communications. They also illustrate complexity of graphene plasmonic responses. Our near-field method provides the possibility for investigations of the surface plasmon phenomena on graphene samples excited by free-space $\mathrm{THz}$ waves. It also allows for characterization of graphene transmission properties with the sub-wavelength spatial resolution.

\section{Acknowledgments}

This work was supported by the Royal Society [Grant No. UF080745] and performed at UCL and at the Center for Integrated Nanotechnologies, an Office of Science User Facility operated for the U.S. Department of Energy (DOE) Office of Science by SNL [Contract No. DE-AC04-94AL85000]. Epitaxial graphene growth and device fabrication are carried out at GaTech, supported by the NSF [DMR-0820382] and the DOE Office of Basic Energy Sciences through a contract with SNL. The work at SNL was supported by a Laboratory Directed Research and Development project. Sandia National Laboratories is a multi-program laboratory managed and operated by Sandia Corporation, a wholly owned subsidiary of Lockheed Martin Corporation, for the U.S. Department of Energy's National Nuclear Security Administration under contract DE-AC04-94AL85000.

\section{References}

1. A. N. Grigorenko, M. Polini, and K. S. Novoselov, Nat Photon 6 (2012) 749-758.

2. M. Jablan, H. Buljan, and M. Soljačić, Physical Review B 80 (2009) 245435.

3. J. M. Poumirol, W. Yu, X. Chen, C. Berger, W. A. de Heer, M. L. Smith, T. Ohta, W. Pan, M. O. Goerbig, D. Smirnov, and Z. Jiang, Physical Review Letters 110 (2013) 246803.

4. L. Ju, B. Geng, J. Horng, C. Girit, M. Martin, Z. Hao, H. A. Bechtel, X. Liang, A. Zettl, Y. R. Shen, and F. Wang, Nat Nano 6 (2011) 630-634.

5. H. Yan, X. Li, B. Chandra, G. Tulevski, Y. Wu, M. Freitag, W. Zhu, P. Avouris, and F. Xia, Nat Nano 7 (2012) 330-334.

6. W. Gao, G. Shi, Z. Jin, J. Shu, Q. Zhang, R. Vajtai, P. M. Ajayan, J. Kono, and Q. Xu, Nano Letters 13 (2013) 3698-3702.

7. I. Crassee, M. Orlita, M. Potemski, A. L. Walter, M. Ostler, T. Seyller, I. Gaponenko, J. Chen, and A. B. Kuzmenko, Nano Letters 12 (2012) 2470-2474.

8. J. H. Strait, P. Nene, W.-M. Chan, C. Manolatou, S. Tiwari, F. Rana, J. W. Kevek, and P. L. McEuen, Physical Review B 87 (2013) 241410.

9. H. Yan, T. Low, W. Zhu, Y. Wu, M. Freitag, X. Li, F. Guinea, P. Avouris, and F. Xia, Nat Photon 7 (2013) 394-399.

10. V. W. Brar, M. S. Jang, M. Sherrott, S. Kim, J. J. Lopez, L. B. Kim, M. Choi, and H. Atwater, Nano Letters 14 (2014) 3876-3880.

11. H. Yan, Z. Li, X. Li, W. Zhu, P. Avouris, and F. Xia, Nano Letters 12 (2012) 3766-3771.

12. J. Chen, M. Badioli, P. Alonso-Gonzalez, S. Thongrattanasiri, F. Huth, J. Osmond, M. Spasenovic, A. Centeno, A. Pesquera, P. Godignon, A. Zurutuza Elorza, N. Camara, F. J. G. de Abajo, R. Hillenbrand, and F. H. L. Koppens, Nature 487 (2012) 77-81.

13. Z. Fei, A. S. Rodin, G. O. Andreev, W. Bao, A. S. McLeod, M. Wagner, L. M. Zhang, Z. Zhao, M. Thiemens, G. Dominguez, M. M. Fogler, A. H. C. Neto, C. N. Lau, F. Keilmann, and D. N. Basov, Nature 487 (2012) 82-85.

14. F. Keilmann and S. Amarie, J Infrared Milli Terahz Waves 33 (2012) 479-484.

15. F. Bonaccorso, Z. Sun, T. Hasan, and A. C. Ferrari, Nat Photon 4 (2010) 611-622.

16. F. Rana, Nanotechnology, IEEE Transactions on 7 (2008) 91-99. 
17. V. Ryzhii, A. A. Dubinov, T. Otsuji, V. Mitin, and M. S. Shur, Journal of Applied Physics 107 (2010) 054505.

18. H. Choi, F. Borondics, D. A. Siegel, S. Y. Zhou, M. C. Martin, A. Lanzara, and R. A. Kaindl, Applied Physics Letters 94 (2009) 172102.

19. M. J. Paul, J. L. Tomaino, J. W. Kevek, T. DeBorde, Z. J. Thompson, E. D. Minot, and Y.-S. Lee, Applied Physics Letters 101 (2012) 091109.

20. J. D. Buron, D. H. Petersen, P. Bøggild, D. G. Cooke, M. Hilke, J. Sun, E. Whiteway, P. F. Nielsen, O. Hansen, A. Yurgens, and P. U. Jepsen, Nano Letters 12 (2012) 5074-5081.

21. O. Mitrofanov, W. Yu, R. J. Thompson, Y. Jiang, I. Brener, W. Pan, C. Berger, W. A. de Heer, and Z. Jiang, Applied Physics Letters 103 (2013) 111105.

22. O. Mitrofanov, M. Lee, J. W. P. Hsu, I. Brener, R. Harel, J. F. Federici, J. D. Wynn, L. N. Pfeiffer, and K. W. West, Selected Topics in Quantum Electronics, IEEE Journal of 7 (2001) 600-607.

23. R. Mueckstein, C. Graham, C. Renaud, A. Seeds, J. Harrington, and O. Mitrofanov, J Infrared Milli Terahz Waves 32 (2011) 1031-1042.

24. W. A. de Heer, C. Berger, M. Ruan, M. Sprinkle, X. Li, Y. Hu, B. Zhang, J. Hankinson, and E. Conrad, Proceedings of the National Academy of Sciences 108 (2011) 16900-16905.

25. C. Berger, Z. Song, X. Li, X. Wu, N. Brown, C. Naud, D. Mayou, T. Li, J. Hass, A. N. Marchenkov, E. H. Conrad, P. N. First, and W. A. de Heer, Science 312 (2006) 1191-1196.

26. J. Hass, F. Varchon, J. E. Millán-Otoya, M. Sprinkle, N. Sharma, W. A. de Heer, C. Berger, P. N. First, L. Magaud, and E. H. Conrad, Physical Review Letters 100 (2008) 125504.

27. M. L. Sadowski, G. Martinez, M. Potemski, C. Berger, and W. A. de Heer, Physical Review Letters 97 (2006) 266405.

28. M. Natrella, O. Mitrofanov, R. Mueckstein, C. Graham, C. C. Renaud, and A. J. Seeds, Opt. Express 20 (2012) 16023-16031.

29. R. Mueckstein and O. Mitrofanov, Opt. Express 19 (2011) 3212-3217.

30. Y. Zhou, X. Xu, F. Hu, X. Zheng, W. Li, P. Zhao, J. Bai, and Z. Ren, Applied Physics Letters 104 (2014) 051106. 